\title{
Occult stress fracture of the thigh
}

\author{
Maximilian Ziernhöld ${ }^{1 *}$ and Franz A Fellner ${ }^{1,2}$ \\ ${ }^{1}$ Central Radiology Institute, Kepler University Hospital, Medical Faculty of the Johannes Kepler University, Linz, Austria \\ ${ }^{2}$ Medical Faculty of the Friedrich-Alexander-University of Erlangen-Nürnberg, Erlangen, Germany
}

\section{Case report}

We report a very sporty, 50 year old professional fireman. A year ago he started training with up to $50 \mathrm{~km}$ of running a week. A month ago there was a contusion of the left knee joint during a fall from the treadmill. Since then, the patient has suffered pain in the area of the lateral thigh compartment, which radiated in the direction of the tibia plateau as well as distal to it and in the area of the lateral ligament attachment. There was no pain in side stretching. Neither valgization nor varification was painful.

Further clinical examination showed a slight varus position of the knee joint and a slightly increased fascia tone of the iliotibial tract. Flexion in the knee caused pain to radiate into the fossa poplitea. The Lasegue test was negative.

The conventional X-ray examination showed an unremarkable finding. A further MR examination was then carried out under the clinical suspicion of a posterior horn lesion of the outer meniscus. In this MR examination, the proton density weighted sequences with fat saturation showed pronounced spotty signal alterations in the distal femur. Therefore, the examination area was extended to the entire thigh (Figure 1).

This showed a pronounced spotty bone marrow edema of the entire distal half of the thigh, laterodorsally with an interruption of the cortical bone. Massive soft tissue edema and circumscribed fluid retention (Figure 2) were seen perifocal. Due to the low mass effect and the lack of contrast agent enhancement in the soft tissue, a malignant lesion appeared unlikely. Therefore, a stress fracture was primarily assumed.

In addition, the lateral meniscus appeared normal, but a complex rupture of the medial meniscus in the pars intermedia and the cornu posterius was shown. Additionally, a computed tomography (CT) of the thigh was performed to assess the suspicious changes in the bone marrow and soft tissues more precisely. The CT findings confirmed the suspicion of a fracture line in the lateral distal femoral diaphysis with callus formation already beginning (Figure 3 ).

Synopsis of the clinic and imaging findings resulted in the diagnosis of a stress fracture of the distal lateral femoral diaphysis.

A renunciation of sport for at least 2 months was discussed with the patient, although a slight exercise bike training was allowed. The leg was not fully relieved. A calcium-rich diet was recommended and oral analgesics were prescribed if necessary.

3 months later, an examination of the thigh revealed a clinically unremarkable finding.

The patient had already started light running training independently. Due to the meniscus lesion, however, the patient still indicated a limitation in athletic resilience, which is why arthroscopy was indicated by the colleagues from trauma surgery.

The conventional X-ray control performed again showed an unremarkable finding, in particular no fracture line could be seen here either (Figure 4).

A control MR examination was carried out after 6 weeks to rule out a malignant genesis of the bone and soft tissue changes. This showed only residual bone marrow changes, the fracture gap was only minimally delimitable, the extensive soft tissue edema has now been completely absorbed (Figure 5).

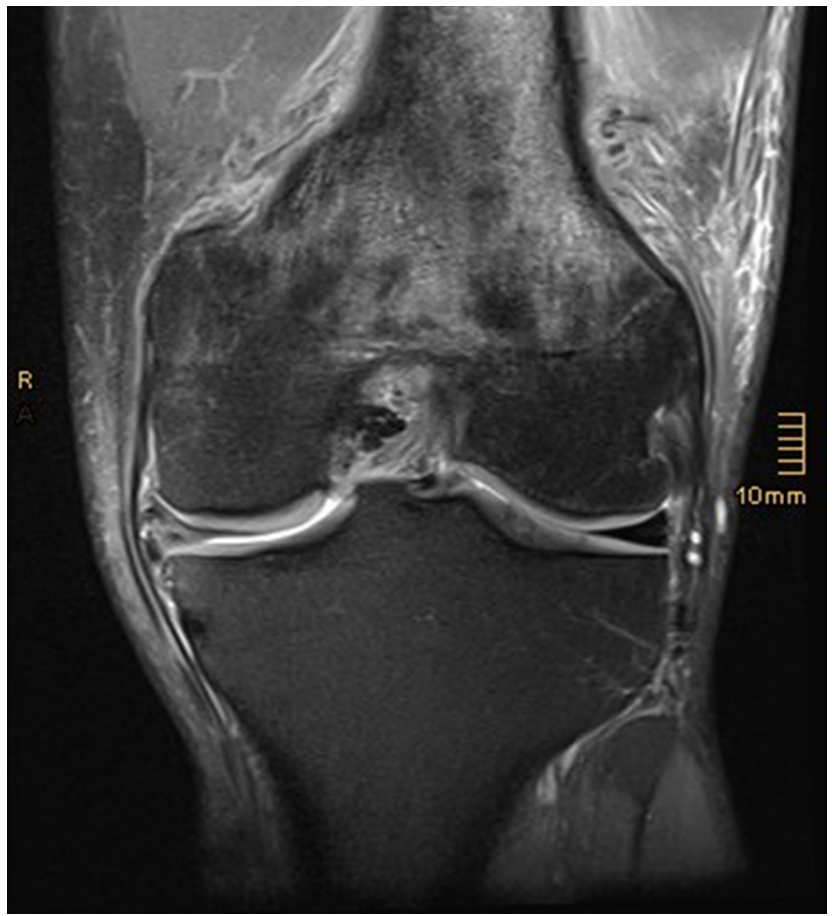

Figure 1. MR imaging: proton-density weighted sequence with fat saturation in coronal orienation. Abnormal signal changes in the bone marrow of the distal femur. Furthermore, complex rupture of the medial meniscus

${ }^{*}$ Correspondence to: Maximilian Ziernhöld, Central Radiology Institute, Kepler University Hospital, Medical Faculty of the Johannes Kepler University, Linz, Austria, E-mail: maximilian.ziernhoeld@kepleruniklinikum.at

Key words: stress fracture, differential diagnosis, upper thigh, musculo-skeletal, magnetic resonance $(\mathrm{mr})$ imaging

Received: May 21, 2020; Accepted: June 17, 2020; Published: June 22, 2020 


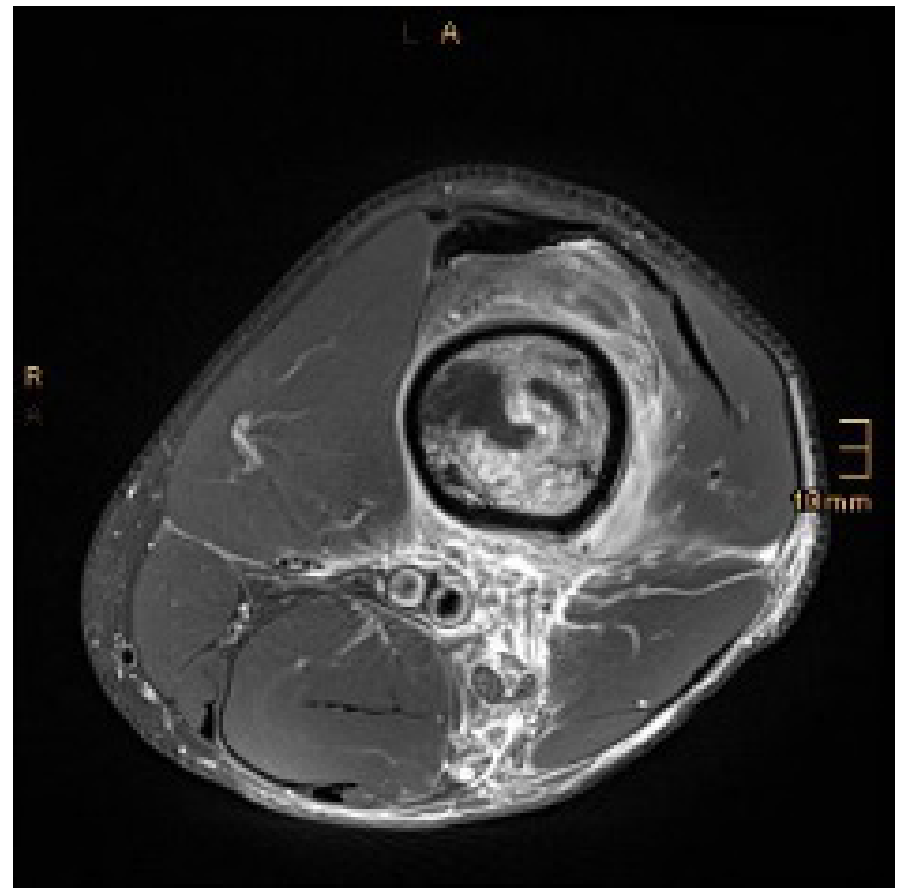

Figure 2. MR imaging of the thigh, proton-density weighted sequence with fat saturation in transverse orientation. This shows suspicious increased signal in the bone marrow and the adjacent soft tissue. Furthermore, there is a discrete linear signal reduction in the bone marrow on the lateral side, which extends to the cortical bone

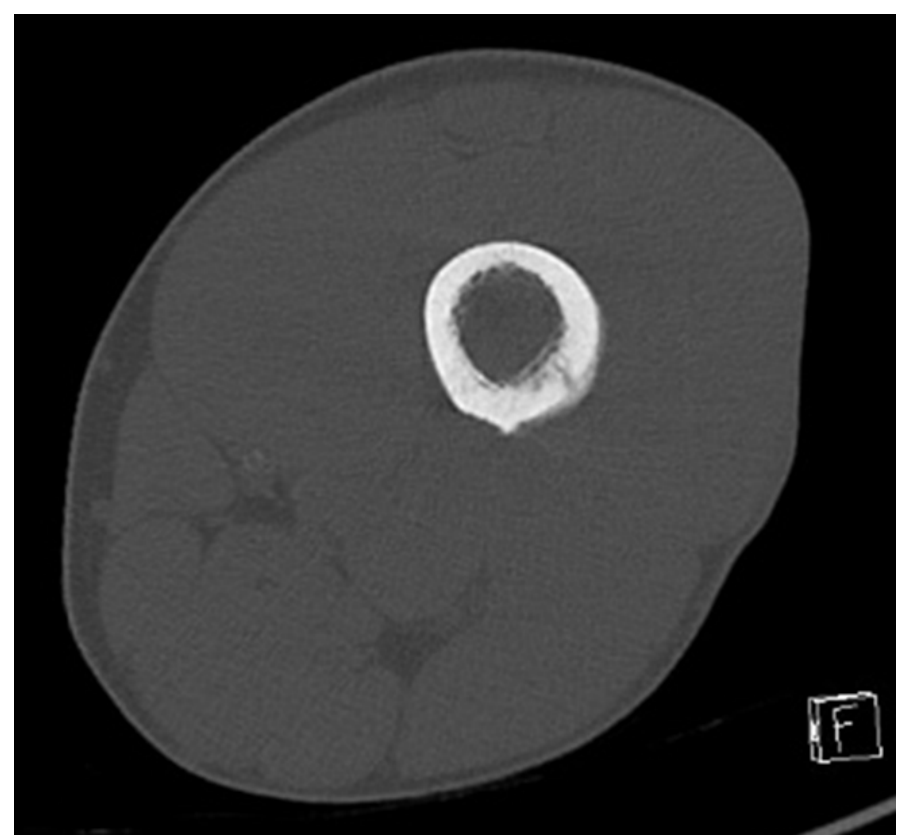

Figure 3. Computed tomography (transverse orientation, bone window) demonstrates incipient callus formation
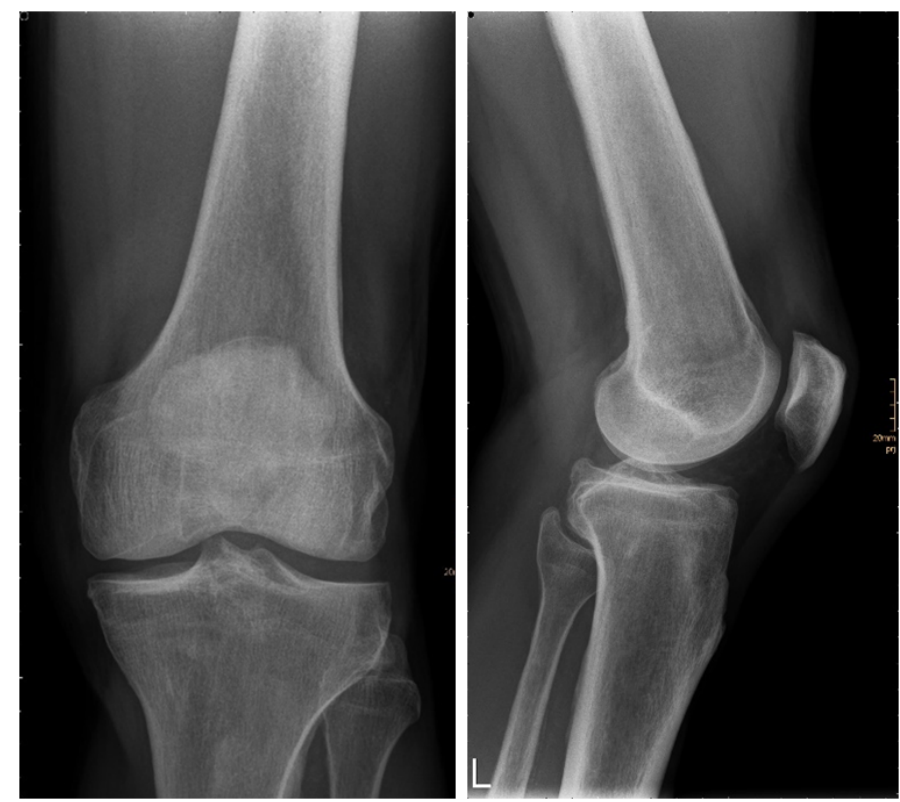

Figure 4. Conventional X-ray control: No proof of fracture

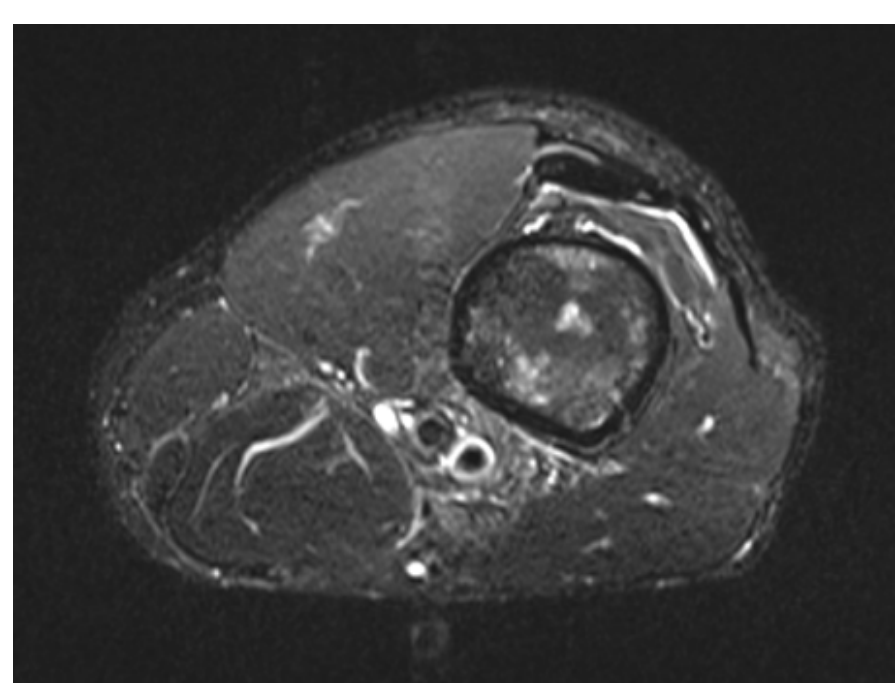

Figure 5. MR control 6 weeks later: transverse STIR sequence reveals only residual bone marrow changes as well as complete remission of soft tissue alteration 


\section{Discussion}

A stress fracture corresponds to a fatigue fracture in healthy bones that occurs below the fracture threshold due to repetitive loads on the normal bone structure [1]. These are often to be found in otherwise healthy people when they start a new physical workout or increase their workout intensity [2]. These fractures occur most frequently in competitive and endurance athletes. In principle, every bone can be affected, but in practice the bones that are exposed to the highest stress during sporting activities, that is, those of the lower extremity, are predestined [3]. The classic anamnesis plays a central role in the diagnosis. In the early phase, the findings are usually unremarkable, there is a creeping, increasing pain, primarily during exercise, then also at rest.

The conventional X-ray examination is the imaging method of choice. However, as with our patient, it is often negative in the early stages with a sensitivity of $15 \%$ [4]. With a suitable medical history and typical course, further clarification is carried out using CT or MRI - in both modalities, the diagnosis is usually successful. However, especially in MR imaging there are frequent misjudgments in the sense of osteoid osteomas, Ewing and osteosarcomas, but also osteomyelitis [1]. Due to the precise anamnesis described above and the targeted search for a fracture line in conventional X-rays or in $\mathrm{CT}$, these are usually avoidable.

In the early stages, the remodeling processes can be depicted well using skeletal scintigraphy, which shows a sensitivity of approximately $100 \%$ - but with low specificity, since even bone changes such as skeletal tumors, infections, bone necrosis or periostitis can sometimes not be differentiated [4].

There are several grading systems for stress reactions and fractures based on MR imaging [5], the most frequently used in the literature being the one proposed by Fredericson et al [6], this initially developed to grade medial tibial stress injuries but since applied elsewhere (Table 1).

Table 1. Grading system for stress reactions and fractures (Fredericson et al. 1995)

\begin{tabular}{|l|l|}
\hline Grade 1 Periosteal edema with no associated bone marrow abnormalities
\end{tabular}

Grade 2 Periosteal edema with mild bone marrow edema visible only on T2-weighted

Grade 2 images with fat suppression

Grade 3 Periosteal edema and extensive bone marrow edema visible on T1-weighted

Grade $3 \begin{aligned} & \text { Periosteal edema and extensive bone marrow edema } \\ & \text { images and T2-weighted images with fat suppression }\end{aligned}$

Grade Periosteal edema, extensive bone marrow edema visible on T1-weighted $\begin{array}{cl}\text { Grade } & \text { images and T2-weighted images with fat suprression and multiple focal areas of } \\ \text { intracortical signal changes }\end{array}$

Grade Periosteal edema, extensive bone marrow edema visible on T1-weighted images

Grade
$\mathbf{4 b}$ and T2-weighted images with fat suprression and linear region of intracortical

4b $\begin{aligned} & \text { and } \mathrm{T} 2 \text {-weighted images withal change (fracture line) } \\ & \text { signal }\end{aligned}$
Robertson et al. propose a subdivision into "high risk fractures" and "low risk fractures" in order to standardize the therapeutic procedure [7]. High risk fractures show an increased risk of fracture propagation, delayed bone fracture healing or the development of non-union. The fractures are localized in zones of increased tensile stress or reduced blood flow, the most common of which are the anterior tibial diaphysis, the metatarsal bone $\mathrm{V}$, the medial malleolus, the lateral femoral neck, and the navicular bone $[8,9]$.

Low risk fractures primarily affect the posteromedial tibia diaphysis, the remaining metatarsal bones, the distal fibula, the medial femoral neck, the calcaneus and the femoral diaphysis.

In most cases, a conservative approach is therapeutically sufficient [5], often reducing or avoiding the stress is sufficient. Relieving measures (crutches) are sometimes carried out. If the course is more severe, the patient must occasionally be immobilized using a bandage or plaster. If conservative treatment is insufficient for a fracture, osteosynthesis may be necessary to prevent the development of non-union.

\section{Conclusion}

Stress fractures do not cause diagnostic difficulties in the case of a typical medical history (gradual onset of symptoms, stress-related pain and usually intense physical exertion). If the symptoms described suggest a different diagnosis than in the patient presented, the primary imaging findings, if they are not conclusive, should in any case lead to further clarification - usually by means of MR imaging - in order to make the correct diagnosis and initiate appropriate therapy.

\section{References}

1. Bath K, Steinhagen I (2019) Stress fracture of athletes as a cause of groin pain. Radiologe 59: 204-211.

2. Geiger J, Rottenburger C, Uhl M (2010) Stress fractures. Radiologie up2date 1: 34-50.

3. Iwamoto J, Sato Y, Takeda T, Matsumoto H (2011) Analysis of stress fractures in athletes based on our clinical experience. World J Orthop 2: 7-12. [Crossref]

4. Freyschmidt J (2015) Stress phenomena on the skeleton. In: Skelettal diseases. 4th edition, Springer, Heidelberg, pp 46-65.

5. Yao L, Johnson C, Gentili A (1998) Stress injuries of bone: analysis of MR imaging staging criteria. Acad Radiol 5: 34-40.

6. Fredericson M, Bergman AG, Hoffman KL, Dillingham MS (1995) Tibial stress reactions in runners: correlation of clinical symptoms and scintigraphy with a new magnetic resonance imaging grading system. Am J Sports Med 23: 472-481. [Crossref]

7. Robertson GA,WoodAM (2017) Lower limb stress fractures in sport: Optimising their management and outcome. World JOrthop 8: 242-255. [Crossref]

8. Behrens SB, Deren ME, Matson A, Fadale PD, Monchik KO (2013) Stress fractures of the pelvis and legs in athletes: a review. Sports Health 5: 165-174. [Crossref]

9. Robertson GA, Wood AM (2015) Return to sports after stress fractures of the tibial diaphysis: a systematic review. Br Med Bull 114: 95-111.

Copyright: (C2020 Ziernhöld M. This is an open-access article distributed under the terms of the Creative Commons Attribution License, which permits unrestricted use, distribution, and reproduction in any medium, provided the original author and source are credited. 